\title{
Analysis of Effectiveness and Performance Prediction of Sports Flipped Classroom Teaching Based on Neural Networks
}

\author{
Wei Xu $\mathbb{D}$, Wenying Xiong, Zhe Shao, and Yun Li $\mathbb{D}$ \\ College of Physical Education and Health, Jiangxi University of Traditional Chinese Medicine, Nanchang 330000, Jiangxi, China \\ Correspondence should be addressed to Yun Li; 20142031@jxutcm.edu.cn
}

Received 28 April 2021; Revised 22 June 2021; Accepted 4 July 2021; Published 31 July 2021

Academic Editor: Chenxi Huang

Copyright $\odot 2021$ Wei Xu et al. This is an open access article distributed under the Creative Commons Attribution License, which permits unrestricted use, distribution, and reproduction in any medium, provided the original work is properly cited.

\begin{abstract}
Traditional physical education methods are unable to meet this requirement due to the practical nature of sports skill teaching. As a result, as the times demanded, the flipped classroom based on neural network technology arose. It has the potential to not only promote the modernization of physical education but also to ensure that it has a positive educational impact. This is a mode of instruction. Furthermore, colleges and universities are increasingly focusing on college students' overall quality development. A method for predicting college students' sports performance using a particle swarm optimization neural network is proposed to accurately predict sports performance and provide a reliable analysis basis for the establishment of sports teaching goals. Neural networks are used in the model. The particle swarm optimization algorithm optimizes the variance and weights of the neural network to improve the accuracy of college students' sports performance predicted by the neural network by updating the particle position and speed through the two extreme values of individual extreme values and global extreme values. Teachers always play the role of the facilitator and helper in the teaching process, which realizes the transformation of teachers' and students' selfpositioning, allows students to better play the lead role, and stimulates students' interest in learning.
\end{abstract}

\section{Introduction}

Flipped classroom [1-3] is a product of modern development, and internet technology is an important support for its implementation: teachers send teaching videos [4-6] made by themselves or by other teachers on the internet to students via the learning platform [7] so that students can complete preclass learning tasks under the guidance of teachers in the classroom. The flipped classroom is popular in theoretical subjects but is rarely used in physical education [8], which is a practical course. However, the emergence of this teaching model allows for the combination of the internet and the physical education classroom. The flipped classroom is a teaching model that reinvents the traditional classroom process of teaching sports skills, bringing the connection between before, during, and after class closer together, and extending the actual teaching and learning time between teachers and students. PE teachers must create small videos based on the teaching content of each lesson in this mode of instruction. Small video produced should be concise and complete the explanation of a lesson's content within 5 to 10 minutes. The level of each physical education teacher varies, as does the quality of the short video produced.

With the ongoing development and popularization of internet technology [9], students are increasingly relying on the convenience that the internet provides. The introduction of internet-based flipped classrooms will undoubtedly cause a revolution in the educational arena. Both students' learning and teachers' teaching have changed as a result of this revolution. Students' learning processes are more independent, and teachers are better able to teach students based on their aptitude. Individuality is a goal for college students, who want to think for themselves. Following the clarification of the learning objectives, they make full use of their ability to consult and gain a preliminary understanding of materials on their own. They understand and learn sports skills before class through sports online courses and then internalize and apply what they have learned in class to improve learning efficiency [10]. 
Students' mastery of sports skills in physical education cannot be truly realized solely through online video learning. Learning sports skills is a unique learning activity that necessitates physical participation and is inextricably linked to classroom exercises. The flipped classroom teaching model allows students to finish their knowledge learning before class. Classroom is a place for teachers to answer students, to interact with teachers and students, and to apply knowledge. The popularization of the internet makes this teaching model more feasible. The flipped classroom based on the neural network [11-14] will extend students' learning time and learning space and is no longer limited to the fixed weekly class time and place. The traditional PE teaching classroom is flipped to a certain extent, and internet is used for learning before and after class. This teaching mode [15] can organically combine the learning of sports skills with online resources under the internet and improve students' enthusiasm for sports learning to a certain extent. Furthermore, students' performance predictions in the sports flipped classroom have a significant impact on classroom interaction. As a result, based on particle swarm optimization neural networks [16-18], this paper proposes a method for predicting college students' sports performance [19].

Machine learning [20] is the science of studying how to use computers to simulate or realize human learning activities. It is one of the most intelligent and cutting-edge research fields in artificial intelligence. Since the 1980s, machine learning, as a way to realize artificial intelligence [21-25], has aroused widespread interest in the artificial intelligence community. Especially in the past ten years, the research work in the field of machine learning has developed rapidly, and it has become an important part of artificial intelligence. Machine learning is not only used in knowledge-based systems but also widely used in many fields [26-30] such as natural language understanding, nonmonotonic reasoning, machine vision [31-33], and pattern recognition $[31,32]$. Therefore, it is feasible to use machine learning to predict the effect and performance of sports flipped classroom teaching.

Following are the main innovation points of this paper:

(1) This paper investigates the use of neural network technology in the sports flip classroom to improve communication between teachers and students, strengthen interaction and cooperation between the two, provide timely and comprehensive sports skill learning for students, improve students' sports skills, and stimulate students' interest in learning sports skills

(2) A prediction method for college students' sports performance based on the particle swarm optimization neural network is proposed to achieve highefficiency and high-precision prediction of college students' sports performance in order to improve the accuracy of college students' sports performance prediction

\section{Related Work}

The term "flipped classroom" refers to rearranging time inside and outside of the classroom in order to transfer learning decision-making power from the teacher to the student. Students can better display their subjective initiative in this classroom teaching model, and teachers can better play their guiding role. Before class, students study independently by watching video lectures and chatting with classmates via the internet. Communication and exchanges between students and teachers have increased as a result of the internet. Students can learn a variety of online courses outside of the classroom if they have access to the internet. The internet era gave birth to this flipped teaching classroom. It has disrupted the traditional teaching classroom structure and resulted in a slew of educational changes.

Motor skills [34] refer to the human body in the movement to master and effectively complete a special movement of an ability. Some scholars believe that it consists of two parts: one is the description of the rules for performing the action, that is, the procedure of the action, and the other is the actual muscle movement, which gradually becomes precise and coherent through practice and feedback. Physical education is a teaching process that takes physical activity as the means, while the teaching of sports skills needs to improve students' ability of sports skills through physical education. Students must feel the joy of the environment, democratic and harmonious interpersonal relationships, individuality liberation, and the learning environment in order to learn sports skills.

Al Zahrani [35] elaborated on the impact of flipped classrooms on the creative thinking of higher education students. In the study, they recruited students from the School of Education of King Abdulaziz University in Saudi Arabia and conducted group teaching experiments on them. Their research results showed that flipped classrooms can improve students' creativity, especially in knowledge, and master the fluency, flexibility, and novelty aspects. Sajid et al. [36] discussed in their article that flipped classroom student-centered active learning replaces passive learning. This learning method improves critical thinking and application skills, including information retention capacity. Larsson and Nyberg [37] stated that sport is the key to physical education. Schools in Sweden are attempting to incorporate social constructivist knowledge and learning concepts into physical education, with an emphasis on students' motor skills. They discussed how to intervene in students' behavior and how to communicate between students and teachers in the article. They believed that students must participate in the teaching process in order for teachers to gain a better understanding of their students and provide better guidance and assistance in the development of motor skills. 


\section{Methodology}

\subsection{Particle Swarm Neural Network Model}

3.1.1. Neuron Model. In 1943, McCulloch and Pitts [38] used a simple model to express the neuron structure and created an artificial neuron model, which is now known as the "M-P neuron model," as shown in Figure 1.

The following is the calculation equation for the neuron's output in Figure 1:

$$
y=f\left(\sum_{i=1}^{n} w_{i} x_{i}-\theta\right)
$$

where $\theta$ is the activation threshold of the neuron we mentioned earlier and the function $f(\cdot)$ is also called the activation function. As shown in the figure, the function $f(\cdot)$ can be expressed by a step equation, which is activated above the threshold; otherwise, it is inhibited, but this is a bit too crude because the step function is not smooth, discontinuous, and nonderivable, so our more commonly used method is to use the sigmoid function to represent the function $f(\cdot)$.

Figure 2 shows the sigmoid activation function, and its calculation equation is as follows:

$$
f(x)=\frac{1}{1+e^{-x}} .
$$

3.1.2. Particle Swarm Optimization. A particle swarm optimization method to solve the optimization problem is summarized by observing the behavior of birds looking for food. It is necessary to search for particles in the optimized space in order to discover the essence of the optimization problem. The speed of each particle determines the distance and direction of the bird's flight. Every particle has a fitness value, and the fitness value is determined by the optimization. The purpose of all particles in space is to find the optimal particle action. The initial random solution (random particle) of the particle swarm optimization method is continuously iterated to find the optimal solution. The particle "tracks" the two extreme values in each iteration to ensure that the particle itself can be updated. These two extreme values are $t_{\text {best }}$ and $u_{\text {best }}$, respectively. $t_{\text {best }}$ is the optimal solution found by the particle itself, called the individual extreme value, and $u_{\text {best }}$ is the optimal solution found by the entire population, called the global extreme value. The particle position and velocity can be updated by the following equation:

$$
\begin{aligned}
w & =w+x_{1} \times y_{1} \times\left(t_{\text {best }}-\text { pre }\right)+x_{2} \times y_{2} \times\left(u_{\text {best }}-\text { pre }\right), \\
\text { pre } & =\text { pre }+w
\end{aligned}
$$

The velocity of the particle is represented by $w$, the position of the particle itself is represented by pre, the random numbers between $(0,1)$ are $y_{1}$ and $y_{2}$, and the learning factors are $x_{1}$ and $x_{2}$, respectively.

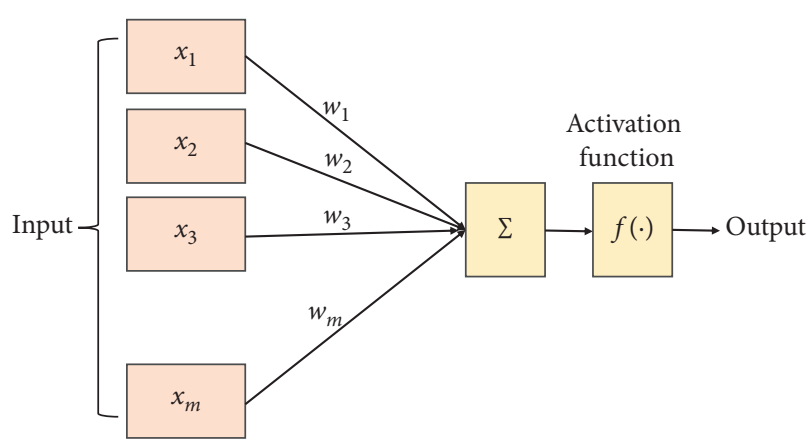

Figure 1: M-P neuron model.

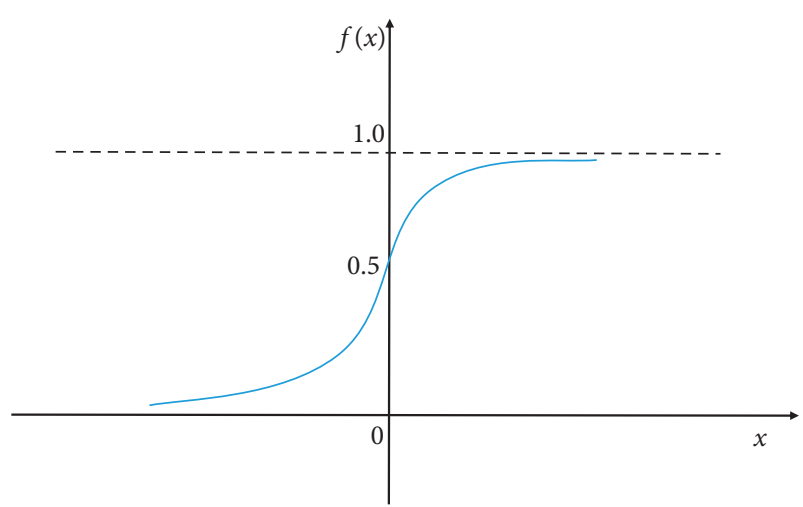

FIGURE 2: Sigmoid function.

Because the first particle velocity $w$ on the right side of equation (3) is random and has no memory, new regions will be explored during the search, demonstrating strong global optimization power. However, in practical applications, it is necessary to conduct a global search first to improve the search's convergence speed and then to conduct a local search to improve the accuracy of the solution obtained. The optimized calculation equation is as follows:

$$
w=\sigma \times w+x_{1}+y_{1} \times\left(t_{\text {best }}-\text { pre }\right)+x_{2} \times y_{2} \times\left(u_{\text {best }}-\text { pre }\right) .
$$

The inertia weight is expressed as $\sigma$. If the value of $\sigma$ is large, the PSO method has a strong global search ability; otherwise, it has a strong local search ability.

3.1.3. Improve Variance and Weights. When using the particle swarm optimization method to optimize the neural network, it is necessary to optimize the variance $\sigma_{i}(i=1,2, \ldots, h)$ and the weight $v_{0}, v_{1}, \ldots, v_{k}$ of the neural network basis function, the dimensions of the two parameters are related to the network structure, and the weight and variance of the neural network are uniformly coded. A set of neural network weights and variances are described by a particle. The root mean square error (RMSE) can be used as a fitness function to reflect the particle's approximation error. The following is the calculation equation for the fitness value of the $i$ th particle: 


$$
\operatorname{RMSE}(i)=\sqrt{\frac{1}{a} \sum_{j=1}^{a}\left(y_{1}^{i}-y_{2}^{i}\right)^{2}},
$$

where $a$ is the total number of samples and the true output value and neural network prediction value are described by $y_{1}^{i}$ and $y_{2}^{i}$, respectively.

\subsection{Effectiveness Analysis and Performance Prediction of the} Sports Flipped Classroom. College students' sports performance is modeled using the particle swarm optimization neural network (PSO) model. In terms of time order, college students' physical education achievements in the past are typical. For the modeling of college students' physical education achievements, a particle swarm optimization neural network model is used. Assume that the initial time series of college students' physical education achievements is shown in the following equation:

$$
A^{(0)}(p)=\left\{a^{(0)}(1), a^{(0)}(2), \ldots, a^{(0)}(m)\right\} .
$$

Operate equation (6) with the first-order differential; then, we can get

$$
A^{(1)}(p)=\left\{a^{(1)}(1), a^{(1)}(2), \ldots, a^{(1)}(m)\right\} .
$$

The first-order mean operation equation (7) can obtain the first-order mean value sequence as follows:

$$
B^{(1)}(p)=\left\{b^{(1)}(1), b^{(1)}(2), \ldots, b^{(1)}(m)\right\} .
$$

Then, a predictive model is established, and the calculation equation is as follows:

$$
\frac{f a^{(1)}}{f p}+c a^{(1)}=\lambda
$$

Solving the above equations, the whitening differential equation that can predict the performance of the sports flipped classroom is as follows:

$$
\widehat{a}^{(1)}(h+1)=\left(a^{(0)}(1)-\frac{\lambda}{c}\right) d^{-c h}+\frac{\lambda}{c} .
$$

Next, the neural network parameters are obtained by training according to the input and output sample data, and the optimal target mapping of equation (10) is completed.

\section{Experiments and Results}

4.1. Experimental Setup. The subjects of the experiment are the two male classes of the 2018 physical education training major of the School of Sports Science of Qufu Normal University-the general volleyball class (17 people). Before determining the experimental objects, preexperiment interviews and physical fitness tests are conducted for the two classes of students. There are significant differences, students who have a foundation in volleyball and other students in the class also participate in the learning, and the data obtained from the learning results are not included in the experiment.
The final students who actually participated in the experiment are listed and determined. Both the experimental class and the control class were of 16 students. The students participating in the teaching experiment are all second-year university students. When students are exposed to new things, they have a strong curiosity about them, as well as strong learning and accepting abilities. Students use mobile phones, computers, and iPads for entertainment, learning, and other purposes in their daily lives. As a result, there will be no problem with them using the school's online program, and there will be no shortage of smart devices and technology.

From March 4 to June 21, a teaching experiment was conducted. Professional teachers provided guidance throughout the process, which included discussing teaching progress with the teacher and carrying out the teaching experiment with the teacher's consent. In the teaching experiment, both the experimental class and the control class are taught twice a week. Each class is two hours. One of the two hours is used to complete experimental teaching, and the remaining one is used to complete the school's teaching plan. The total class hours for teaching experiments are 32 hours.

4.2. Experimental Results. Start at midpoint $A$ of the end line, and the stopwatch starts at the moment of start. Run along line 1 to intersection point $B$ of the end line and the sideline, touch the volleyball, and then quickly turn back to midpoint $A$ of the end line. Touch the volleyball, and then quickly run along line 2 to intersection point $C$ of the offensive line and the sideline, touch the volleyball, and then quickly follow 2 . Fold back to midpoint $A$ of the end line, touch the volleyball, and then quickly run to midpoint $D$ of the offensive line along the 3rd line. Touch the volleyball, continue to fold back to midpoint $A$ of the end line along the 3 rd line, and then run along the 4 th line towards the offensive line. Touch the volleyball at intersection point $E$ of the sideline, and then fold back to midpoint $A$ of the end line along line 4 . Touch the volleyball, finally run along line 5 to intersection point $F$ between the end line and the sideline, fold back to point $A$, complete the half-meter run test, and stop timing. At six points $A, B, C, D, E$, and $F$, arrange a student to place a volleyball, and supervise the tester to touch the volleyball during the movement. The moving route is shown in Figure 3. In addition, Figure 4 also gives the experimental scene of the physical fitness test.

Table 1 shows that the neural network-based method used in this article outperforms the traditional method significantly. BP network [33] is slightly inferior to the other two neural network methods. As a result, the algorithm presented in this article is capable of analyzing and predicting the effectiveness of sports flipped classroom teaching.

It can be seen from Figure 5 that when the sports flipped classroom performance predicted by this method is used, the sports performance prediction accuracy of each sports item is higher than 9500, while the accuracy of the other two methods is below $90 \%$ for both, indicating that the sports 


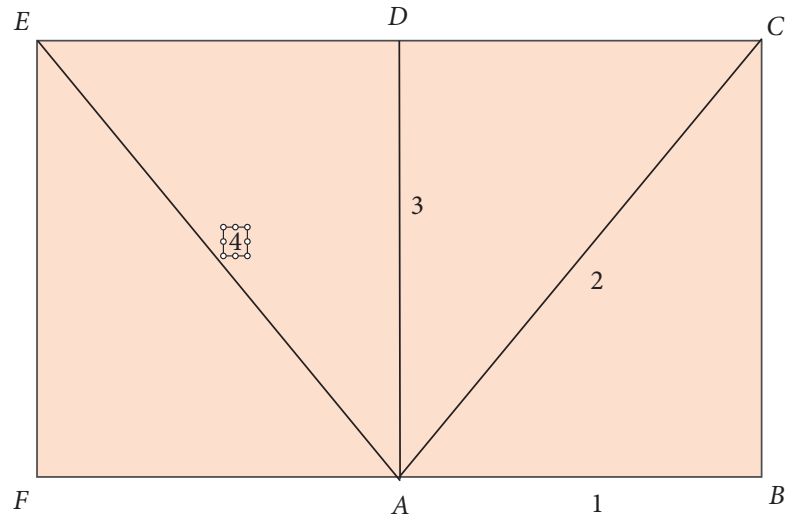

Figure 3: Experimental site.

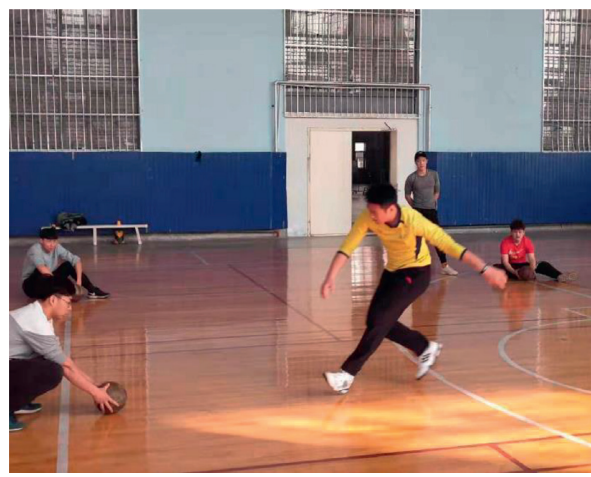

(a)

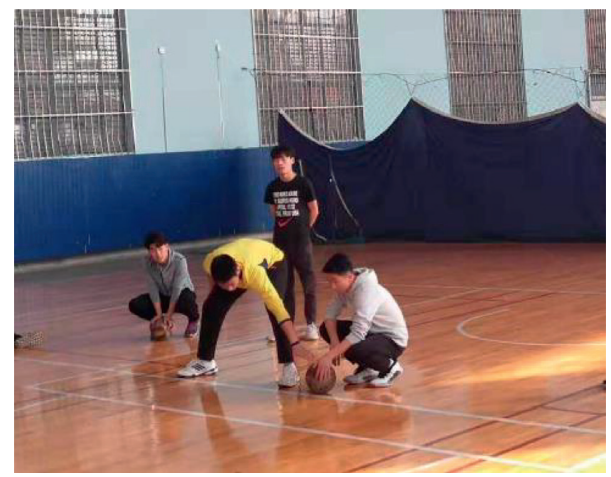

(b)

Figure 4: Physical fitness test.

TABle 1: Experimental results of the speed test.

\begin{tabular}{lcccc}
\hline Method & $N$ & Mean & SD & Acc \\
\hline Traditional & 16 & 18.11 & 0.77 & 0.81 \\
BP & 16 & 18.12 & 0.81 & 0.85 \\
Hinojo-Lucena et al. & 16 & 18.62 & 0.94 & 0.92 \\
Ours & 16 & 18.63 & 0.96 & 0.92 \\
\hline
\end{tabular}

flipping classroom performance prediction of the method in this paper has strong versatility and high precision and can be widely used in the future study of college students' sports performance.

4.3. Ablation Experiment. We conducted an ablation experiment. We input 3, 5, and 8 factors to observe their influence on the experimental results. The experimental results are shown in Table 2.

It can be clearly seen from Table 2 that, with the increase of factors, the model's prediction accuracy rate increases, but at the same time, we found that, from 5 factors to 8 factors, the accuracy rate has not greatly improved, considering the collection of data, and preprocessing can be a tedious work; therefore, this article believes that it is more appropriate to consider five factors.

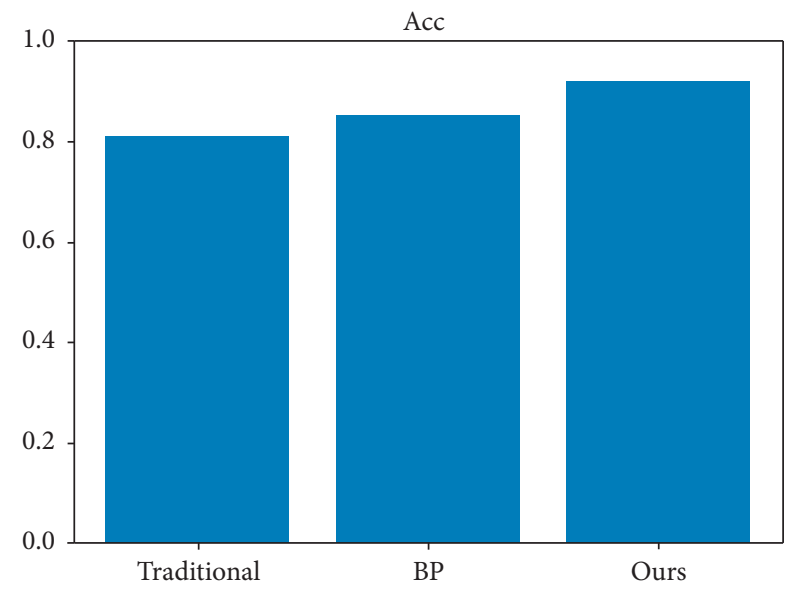

FIgURE 5: Experimental results of accuracy comparison.

TABLE 2: Results of ablation experiments.

\begin{tabular}{lc}
\hline Input & Acc \\
\hline 3 factors & 0.8636 \\
5 factors & 0.9207 \\
8 factors & 0.9258 \\
\hline
\end{tabular}




\section{Conclusion}

To accurately predict sports performance and provide a reliable analysis basis for the establishment of sports teaching goals, a method for predicting college students' sports performance based on the particle swarm optimization neural network is proposed. The model is based on neural networks. The particle swarm optimization algorithm updates the particle position and speed through the two extreme values of individual extreme values and global extreme values to optimize the variance and weights of the neural network to enhance the accuracy of college students' sports performance predicted by the neural network. In the teaching process, teachers always take the role of the facilitator and helper to carry out teaching, which realizes the transformation of teachers' and students' self-positioning, can better play the main role of students, and stimulate students' interest in learning.

\section{Data Availability}

The data used to support the findings of this study are included within the article.

\section{Conflicts of Interest}

All the authors declare no conflicts of interest.

\section{References}

[1] G. Akçayır and M. Akçayır, "The flipped classroom: a review of its advantages and challenges," Computers \& Education, vol. 126, pp. 334-345, 2018.

[2] K. F. Hew and C. K. Lo, "Flipped classroom improves student learning in health professions education: a meta-analysis," BMC Medical Education, vol. 18, no. 1, pp. 38-12, 2018.

[3] P. Strelan, A. Osborn, and E. Palmer, "The flipped classroom: a meta-analysis of effects on student performance across disciplines and education levels," Educational Research Review, vol. 30, Article ID 100314, 2020.

[4] S. L. Cresswell, W. A. Loughlin, M. J. Coster, and D. M. Green, "Development and production of interactive videos for teaching chemical techniques during laboratory sessions," Journal of Chemical Education, vol. 96, 2019.

[5] A. Meseguer-Martinez, A. Ros-Galvez, and A. Rosa-Garcia, "Satisfaction with online teaching videos: a quantitative approach," Innovations in Education \& Teaching International, vol. 54, no. 1, pp. 62-67, 2017.

[6] P. Born, N. P. Nguyen, R. Grambow, D. Meffert, and T. Vogt, "Embedding tennis-specific teaching videos into long-term educational concepts to improve movement learning and technique performances," Journal of Physical Education and Sport, vol. 18, no. 1, pp. 255-261, 2018.

[7] N. F. B. Casillano, "Challenges of implementing an E-learning platform in an Internet struggling province in the Philippines," Indian Journal of Science and Technology, vol. 12, no. 10, pp. 1-4, 2019.

[8] A. Casey and A. MacPhail, "Adopting a models-based approach to teaching physical education," Physical Education and Sport Pedagogy, vol. 23, no. 3, pp. 294-310, 2018.

[9] A. Zohrabi, J. Karimi, and A. Mohebi, "Explaining the applications of Internet of things in Iran's sports industry: a sustainable development approach," Communication Management in Sport Media, vol. 7, no. 3, pp. 91-99, 2020.

[10] T. Tang, A. M. Abuhmaid, M. Olaimat, D. M. Oudat, M. Aldhaeebi, and E. Bamanger, "Efficiency of flipped classroom with online-based teaching under COVID-19," Interactive Learning Environments, pp. 1-12, 2020, In press.

[11] J. Zhang, Y. Liu, H. Liu, and J. Wang, "Learning local-global multiple correlation filters for robust visual tracking with kalman filter redetection," Sensors, vol. 21, no. 4, Article ID 1129, 2021.

[12] W. Cai and Z. Wei, "Remote sensing image classification based on a cross-attention mechanism and graph convolution," IEEE Geoscience and Remote Sensing Letters, 2020, In press.

[13] X. Ning, X. Wang, and $\mathrm{S} \mathrm{Xu,} \mathrm{"A} \mathrm{review} \mathrm{of} \mathrm{research} \mathrm{on} \mathrm{co-}$ training. concurrency and computation: practice and experience," Concurrency and Computation Practice and Experience, 2021, In press.

[14] W. Cai, B. Liu, Z. Wei, M. Li, and J. Kan, "TARDB-Net: tripleattention guided residual dense and BiLSTM networks for hyperspectral image classification," Multimedia Tools and Applications, vol. 80, pp. 1-22, 2021.

[15] Q. Luo, "Research on the teaching mode of physical education in colleges and universities," in Proceedings of the 2017 2nd International Conference on Education, Sports, Arts and Management Engineering (ICESAME 2017), pp. 716-719, Zhengzhou, China, June 2017.

[16] Y. Zhang, W. Li, L. Zhang, X. Ning, L. Sun, and Y. Lu, "AGCNN: adaptive gabor convolutional neural networks with receptive fields for vein biometric recognition," Concurrency and Computation: Practice and Experience, Article ID e5697, 2020, In press.

[17] W. Cai, Y. Song, and Z. Wei, "Multimodal data guided spatial feature fusion and grouping strategy for e-commerce commodity demand forecasting," Mobile Information Systems, vol. 2021, Article ID 5568208, 14 pages, 2021.

[18] X. Zhang, Y. Yang, Z. Li, X. Ning, Y. Qin, and W. Cai, “An improved encoder-decoder network based on strip pool method applied to segmentation of farmland vacancy field," Entropy, vol. 23, no. 4, p. 435, 2021.

[19] F. Muñoz-Bullón, M. J. Sanchez-Bueno, and A. Vos-Saz, "The influence of sports participation on academic performance among students in higher education," Sport Management Review, vol. 20, no. 4, pp. 365-378, 2017.

[20] C. Yan, G. Pang, X. Bai et al., "Beyond triplet loss: person reidentification with fine-grained difference-aware pairwise loss," IEEE Transactions on Multimedia, 2021, In press.

[21] X. Ning, Y. Wang, W. Tian, L. Liu, and W. Cai, "A biomimetic covering learning method based on principle of homology continuity," ASP Transactions on Pattern Recognition and Intelligent Systems, vol. 1, no. 1, pp. 9-16, 2021.

[22] W. Cai, Z. Wei, R. Liu, Y. Zhuang, Y. Wang, and X. Ning, "Remote sensing image recognition based on multi-attention residual fusion networks," ASP Transactions on Pattern Recognition and Intelligent Systems, vol. 1, no. 1, pp. 1-8, 2021.

[23] X. Ning, K. Gong, W. Li, L. Zhang, X. Bai, and S. Tian, "Feature refinement and filter network for person Re-identification," IEEE Transactions on Circuits and Systems for Video Technology, 2020, In press.

[24] J. Zhang, J. Sun, J. Wang, and X. G. Yue, "Visual object tracking based on residual network and cascaded correlation filters," Journal of Ambient Intelligence and Humanized Computing, pp. 1-14, 2020, In press. 
[25] X. Ning, P. Duan, W. Li, and S. Zhang, "Real-time 3D face alignment using an encoder-decoder network with an efficient deconvolution layer," IEEE Signal Processing Letters, vol. 27, pp. 1944-1948, 2020.

[26] J. Zhang, X. Jin, J. Sun, J. Wang, and K. Li, "Dual model learning combined with multiple feature selection for accurate visual tracking," IEEE Access, vol. 7, pp. 43956-43969, 2019.

[27] X. Ning, K. Gong, W. Li, and L. Zhang, "JWSAA: joint weak saliency and attention aware for person re-identification," Neurocomputing, vol. 453, pp. 801-811, 2020.

[28] R. Liu, X. Ning, W. Cai, and G. Li, "Multiscale dense crossattention mechanism with covariance pooling for hyperspectral image scene classification," Mobile Information Systems, vol. 2021, Article ID 9962057, 15 pages, 2021.

[29] Y. Jiang, X. Gu, D. Wu et al., "A novel negative-transferresistant fuzzy clustering model with a shared cross-domain transfer latent space and its application to brain CT image segmentation," IEEE/ACM Transactions on Computational Biology and Bioinformatics, vol. 18, no. 1, pp. 40-52, 2020.

[30] Y. Gu, A. Chen, X. Zhang, C. Fan, K. Li, and J. Shen, "Deep learning based cell classification in imaging flow cytometer," ASP Transactions on Pattern Recognition and Intelligent Systems, vol. 1, no. 2, pp. 18-27, 2021.

[31] Y. Jiang, K. Zhao, and K Xia, "A novel distributed multitask fuzzy clustering algorithm for automatic MR brain image segmentation," Journal of Medical Systems, vol. 43, no. 5, pp. 118-119, 2019.

[32] Z. Huang, P. Zhang, R. Liu, and D. Li, "Immature apple detection method based on improved Yolov3," ASP Transactions on Internet of Things, vol. 1, no. 1, pp. 9-13, 2021.

[33] L. Huang, G. Xie, W. Zhao, Y. Gu, and Y. Huang, "Regional logistics demand forecasting: a bp neural network approach," Complex \& Intelligent Systems, pp. 1-16, 2021, In press.

[34] S. W. Logan, S. M. Ross, K. Chee, D. F. Stodden, and L. E. Robinson, "Fundamental motor skills: a systematic review of terminology," Journal of Sports Sciences, vol. 36, no. 7, pp. 781-796, 2018.

[35] A. M. Al-Zahrani, "From passive to active: the impact of the flipped classroom through social learning platforms on higher education students' creative thinking," British Journal of Educational Technology, vol. 46, no. 6, pp. 1133-1148, 2015.

[36] M. R. Sajid, A. F. Laheji, F. Abothenain, Y. Salam, D. AlJayar, and A. Obeidat, "Can blended learning and the flipped classroom improve student learning and satisfaction in Saudi Arabia?" International Journal of Medical Education, vol. 7, pp. 281-285, 2016.

[37] H. Larsson and G. Nyberg, "'It doesn't matter how they move really, as long as they move.' Physical education teachers on developing their students' movement capabilities," Physical Education and Sport Pedagogy, vol. 22, no. 2, pp. 137-149, 2017.

[38] W. S. McCulloch and W. Pitts, "A logical calculus of the ideas immanent in nervous activity," Bulletin of Mathematical Biophysics, vol. 5, no. 4, pp. 115-133, 1943. 